\title{
Triangle/Square Waveform Generator Using Area Efficient Hysteresis Comparator
}

\author{
Martin DRINOVSKY ${ }^{1,2}$, Jiri HOSPODKA ${ }^{1}$ \\ ${ }^{1}$ Dept. of Circuit Theory, Czech Technical University in Prague, Technická 2, 16627 Praha, Czech Republic \\ ${ }^{2}$ STMicroelectronics Design and Application s.r.o., Pobřežní 620/3, 18600 Praha, Czech Republic \\ martin.drinovsky@st.com, hospodka@fel.cvut.cz
}

Manuscript received November 16, 2015

\begin{abstract}
A function generator generating both square and triangle waveforms is proposed. The generator employs only one low area comparator with accurate hysteresis set by a bias current and a resistor. Oscillation frequency and its non-idealities are analyzed. The function of the proposed circuit is demonstrated on a design of $1 \mathrm{MHz}$ oscillator in STMicroelectronics $180 \mathrm{~nm}$ BCD technology. The designed circuit is thoroughly simulated including trimming evaluation. It consumes $4.1 \mu \mathrm{A}$ at $1.8 \mathrm{~V}$ and takes $0.0126 \mathrm{~mm}^{2}$ of silicon area. The temperature variation from $-40^{\circ} \mathrm{C}$ to $125^{\circ} \mathrm{C}$ is $\pm 1.5 \%$ and the temperature coefficient is $127 \mathrm{ppm} /{ }^{\circ} \mathrm{C}$.
\end{abstract}

\section{Keywords}

Function generator, triangle and square wave generator, hysteresis comparator

\section{Introduction}

Relaxation oscillator circuits are present in almost every electronic application such as microcontrollers, DC-DC converters or RFID chips. With increasing demand for small form surface mount packages silicon area of every block can have an impact on whether the final design fits into the package or not. Recently several architectures with low silicon area have been proposed. In [1] a capacitor is linearly charged and the threshold is compared with a current-mode comparator. In [2] a voltage on an exponentially charged capacitor is compared with a hysteresis comparator. However, for some applications, such as DC-DC converters [3], a triangular waveform may be required.

The conventional approach to generation of such a waveform is depicted in Fig. 1. A capacitor is charged with a constant current of alternating orientation generated by a current source (CS) between two voltage levels $V_{\text {lo }}$ and $V_{\text {hi }}$. This solution requires two comparators. Instead of two comparators another solutions may employ single comparator with a hysteresis. In [4] a CMOS Schmitt trigger is used.

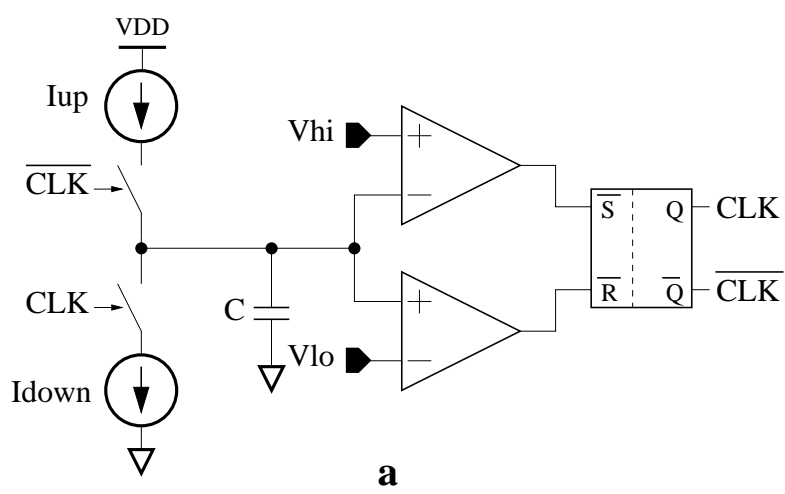

Fig. 1. Conventional triangle oscillator.

The main drawback of such a circuit is that the hysteresis and the frequency are sensitive to PVT variations.

To address this issue, the solution in [5] uses a comparator with a hysteresis set by an external resistor network. In [6] the hysteresis is set with a resistor and a saturation current of an OTA. In [7] two OTAs are used to form a Schmitt trigger and another OTA is used as an integrator. These solutions require either comparator or OTA with a differential input stage.

Another class of generators is based on the so-called modern functional blocks. In [8] two second-generation current conveyors (CCII) are used for square/triangular generation. A current mode generator is presented in [9] using two multiple-output current controlled current differencing transconductance amplifiers (MO-CCCDTA). Another voltage mode solution uses two differential voltage current conveyors (DVCC) in [10]. A differential output generation was presented in [11] using dual output and fully balanced voltage differencing buffered amplifiers (DO-VDBA and FB-VDBA, respectively). Solutions in [12] and [13] employ single Zcopy controlled gain voltage differencing current conveyors (ZC-CG-VDCC) for voltage/current output functional generator. All these designs require complex functional blocks that take a lot of silicon area. The overview of the mentioned architectures can be seen in Tab. 1 . 


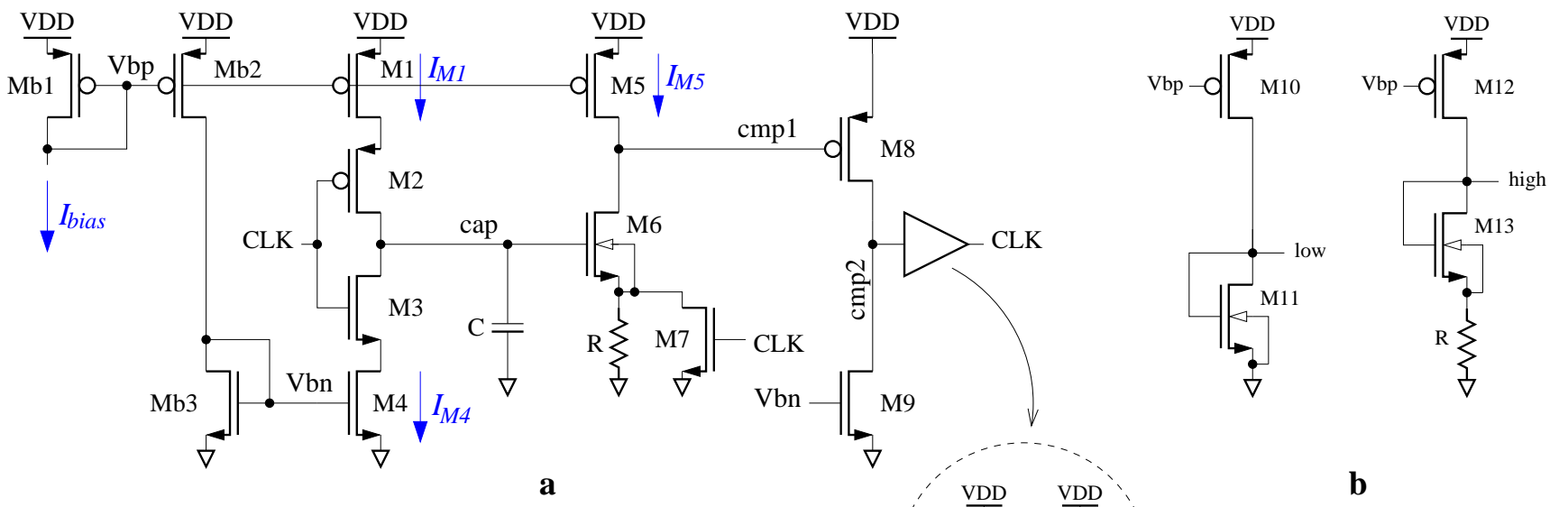

Fig. 2. Proposed circuit: (a) Waveform generator, (b) reference generators (when not explicitly shown the bulks are tied to $V_{\mathrm{DD}}$ or ground for PMOS and NMOS, respectively).

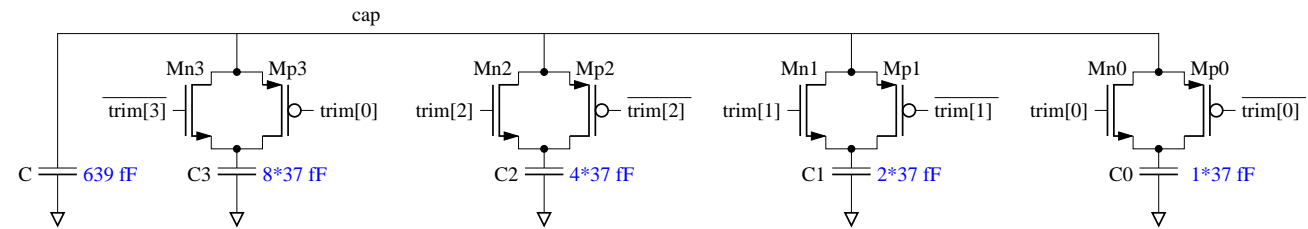

Fig. 3. Trimming circuit.

\begin{tabular}{|c|c|c|c|c|}
\hline 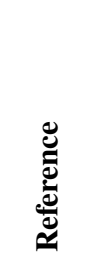 & 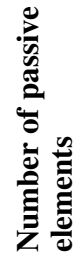 & 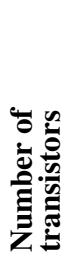 & 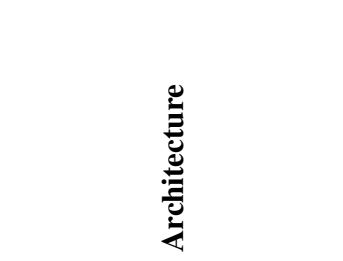 & 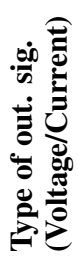 \\
\hline [4] & 1 & 19 & CS + CMOS Schmitt trig. & Volt. \\
\hline [5] & 4 & N/A & CS + Opamp & Volt. \\
\hline [6] & 2 & 15 & CS + OTA & Volt. \\
\hline [7] & 3 & N/A & $3 \times$ OTA & Volt. \\
\hline [8] & 4 & N/A & $2 \times \mathrm{CCII}$ & Volt. \\
\hline [9] & 1 & 58 & $2 \times \mathrm{MO}-\mathrm{CCCDTA}$ & Cur. \\
\hline [10] & 4 & N/A & $2 \times$ DVCC & Volt. \\
\hline [11] & 3 & 24 & DO-VDBA + FB-VDBA & Volt. \\
\hline [12] & 3 & 52 & ZC-CG-VDCC & Both \\
\hline$[13]$ & 1 & N/A & ZC-CG-VDCC & Both \\
\hline Fig. 2a & 2 & 20 & $\mathrm{CS}+$ single input comp. & Volt. \\
\hline
\end{tabular}

Tab. 1. Comparison of selected architectures.

In this article a new triangular relaxation oscillator is proposed. This circuit requires only one single ended comparator and therefore saves both silicon area and power consumption. Section 2 described operation of the proposed circuit and analyses its oscillation frequency, design of a $1 \mathrm{MHz}$ relaxation oscillator can be found in Sec. 3 and its simulation results are presented in Sec. 4, conclusion follows in Sec. 4.

\section{Circuit Analysis}

The schematic of the proposed waveform generator is in Fig. 2a. Transistors $M_{1}$ and $M_{4}$ work as current sources with $M_{2-3}$ as switches controlling charging and discharging of the capacitor $C$. For symmetrical waveform both current must be equal. Hysteresis comparator is composed of transistors $M_{5-9}$. $M_{6}$ together with $R$ work as $V \rightarrow I$ converter whose output current is compared to $I_{M 5}$ produced by $M_{5} . M_{8}$ and $M_{9}$ then form a second stage of the comparator whose output is further amplified by a digital CMOS buffer. Hysteresis is created by shorting the resistor by $M_{7}$. If the bulk of $M_{6}$ is shorted to the source the body effect is avoided and the two threshold voltages are (assuming high gain in the first stage of the comparator):

$$
\begin{aligned}
V_{\text {low }} & =\left.V_{g s 6}\right|_{I_{M 5}}, \\
V_{\text {high }} & =\left.V_{g s 6}\right|_{I_{M 5}}+R I_{M 5} .
\end{aligned}
$$

Absolute value of the two voltages is dependent on the gatesource voltage of $M_{6}$ but the difference depends only on the current in the first stage of the comparator and the resistor value.

Some applications (e.g. DC-DC converters) may require reference voltages corresponding to the comparator thresholds. These can be extracted with the circuits depicted in Fig. 2b. $M_{11}$ is sized to have the same current density as 
$M_{6}$ so that $V_{g s 11}=V_{g s 6}=V_{\text {low }}$. Similarly, if $I_{M 12}=I_{M 5}$ and $M_{13}$ is the same size as $M_{6}$ then the voltage on the drain of $M_{13}$ corresponds to $V_{\mathrm{high}}$. By the same principle, if needed, setting the resistor value between 0 and $R$ (resistor value in the oscillator) can generate any voltage within the oscillator output voltage range.

Due to the delay of the comparator the capacitor voltage $v_{\text {cap }}$ overshoots the threshold $V_{\text {high }}$ by $\mathrm{SR}^{+} t_{d}^{+}$, where $\mathrm{SR}^{+}$is the positive slew rate on the capacitor given by $\frac{I_{M 1}}{C}$ and $t_{d}^{+}$ if the rising propagation delay of the comparator. Similarly for the opposite phase $v_{\text {cap }}$ undershoots $V_{\text {low }}$ by $\mathrm{SR}^{-} t_{d}^{-}, \mathrm{SR}^{-}$ being negative slew rate given by $\frac{I_{M 4}}{C}$ and $t_{d}^{-}$being falling propagation delay. The rising and falling half-periods are

$$
T^{+/-}=\frac{\left(V_{\text {high }}+\mathrm{SR}^{+} t_{d}^{+}\right)-\left(V_{\text {low }}-\mathrm{SR}^{-} t_{d}^{-}\right)}{\mathrm{SR}^{+/-}} .
$$

Substituting (1) and (2) into (3) and summing both halfperiods we get for a symmetrical waveform $\left(\mathrm{SR}^{+}=\mathrm{SR}^{-}\right)$the following oscillation period

$$
T=2\left(R C \frac{I_{M 5}}{I_{M 1}}+t_{d}^{+}+t_{d}^{-}\right) .
$$

The frequency of oscillation is therefore dependent on the product of $R$ and $C$ as is the temperature dependence. The effect of the comparator delay can be compensated by increasing the value of $C$.

The comparator delay portion of the oscillation period is dominated by $t_{d}^{-}$caused mostly by slewing of the $\mathrm{cmpl}$ node from saturation voltage of $M_{6} V_{d s 6}^{\text {sat }}$ to the threshold of the second stage give by $V_{\mathrm{DD}}-\left|V_{\mathrm{THP}}\right|$, where $V_{\mathrm{THP}}$ is the threshold voltage of PMOS transistor $M_{8}$. This delay $t_{\text {slew }}$ can be estimated as follows. Slewing starts when the input voltage of the comparator crosses threshold $V_{\text {low }}$. Around this operating point $M_{6}$ can be approximated by a corresponding transconductance $g_{m}$ that is charging a parasitic capacitance $C_{p}$ of node $\mathrm{cmpl}$. As the voltage on the capacitor $v_{\text {cap }}$ continues to linearly decrease so does the current charging $C_{p}$ given by $g_{m} 6 v_{\text {cap }}$. The slewing time can be computed by the following integral

$$
\begin{aligned}
V_{\mathrm{DD}}-\left|V_{\mathrm{THP}}\right|-V_{d s 6}^{\mathrm{sat}} & =\int_{0}^{t_{\text {slew }}} \frac{g_{m 6} v_{\mathrm{cap}}}{C_{p}} \mathrm{~d} t \\
& =\int_{0}^{t_{\text {slew }}} \frac{g_{m 6} \mathrm{SR}^{-} t}{C_{p}} \mathrm{~d} t .
\end{aligned}
$$

Solving for $t_{\text {slew }}$ leads to

$$
t_{d}^{-} \approx t_{\text {slew }}=\sqrt{\frac{2\left(V_{D D}-\left|V_{\mathrm{THP}}\right|-V_{d s 6}^{\mathrm{sat}}\right) C_{p}}{g_{m 6} \mathrm{SR}^{-}}} .
$$

Equation (6) shows that to decrease the slewing delay of the comparator the parasitic capacitance $C_{p}$ must be minimized and the transconductance $g_{m 6}$ must be maximized. The former can be done by minimizing $M_{8}$ for lower gate capacitance, the latter by increasing drain current of $M_{6}$ which is given by $I_{M 5}$.

\section{Design}

The proposed waveform generator with $1 \mathrm{MHz}$ frequency was designed in STMicroelectronics $180 \mathrm{~nm}$ BCD technology with supply voltage of $1.8 \mathrm{~V}$. Values of the passive components were selected to be easily integrated onchip: $R=500 \mathrm{k} \Omega, C=1 \mathrm{pF}$. For good linearity a MOM (Metal-Oxide-Metal) capacitor was selected together with $\mathrm{N}+$ polysilicon resistor for good temperature behavior.

Since the on-chip resistors and capacitors have large process variations trimming is usually employed to put the resultant frequency within a given specification. This can be accomplished by trimming either the resistor or the capacitor. The drawback of the resistor trimming is a change of triangle amplitude with trimming code. This may not be an issue when only a digital output is used but may pose a problem for subsequent processing when the triangular output is used as well, e.g. in DC-DC converters. For this reason the capacitor trimming was selected and the trimming circuit can be found in Fig. 3. The main capacitor $C$ is accompanied with four binary scaled capacitor $C_{0}-C_{4}$ which can be switched parallel to the main capacitor using transfer gates controlled by trimming bits. The unit capacitance of the trimming capacitors and therefore the trimming range was selected according to the technology spread of the oscillation period and is about $\pm 30 \%$. The remaining value of the main capacitor $C$ was then reduced by the parasitic capacitances of the transistors connected to the cap node, e.g. drain/source capacitances of $M_{n 0-3}, M_{p 0-3}, M_{2}, M_{3}$ or gate capacitance of $M_{6}$. This correction makes $65 \mathrm{fF}$.

\begin{tabular}{|l|c|}
\hline Component & Width / Length \\
\hline$M_{b 1}, M_{b 2}, M_{1}, M_{5}, M_{10}, M_{12}$ & $2 \mu \mathrm{m} / 2 \mu \mathrm{m}$ \\
\hline$M_{b 3}, M_{4}, M_{9}$ & $1 \mu \mathrm{m} / 4 \mu \mathrm{m}$ \\
\hline$M_{2}$ & $2 \mu \mathrm{m} / 0.18 \mu \mathrm{m}$ \\
\hline$M_{3}$ & $1 \mu \mathrm{m} / 0.18 \mu \mathrm{m}$ \\
\hline$M_{6}, M_{11}, M_{13}$ & $2 \mu \mathrm{m} / 0.56 \mu \mathrm{m}$ \\
\hline$M_{7}$ & $1 \mu \mathrm{m} / 0.18 \mu \mathrm{m}$ \\
\hline$M_{8}, M_{d 1}, M_{d 3}$ & $1 \mu \mathrm{m} / 0.24 \mu \mathrm{m}$ \\
\hline$M_{d 2}, M_{d 4}$ & $0.7 \mu \mathrm{m} / 0.24 \mu \mathrm{m}$ \\
\hline$M_{n 0-3}$ & $0.7 \mu \mathrm{m} / 0.18 \mu \mathrm{m}$ \\
\hline$M_{p 0-3}$ & $1 \mu \mathrm{m} / 0.18 \mu \mathrm{m}$ \\
\hline
\end{tabular}

Tab. 2. Transistor dimensions.

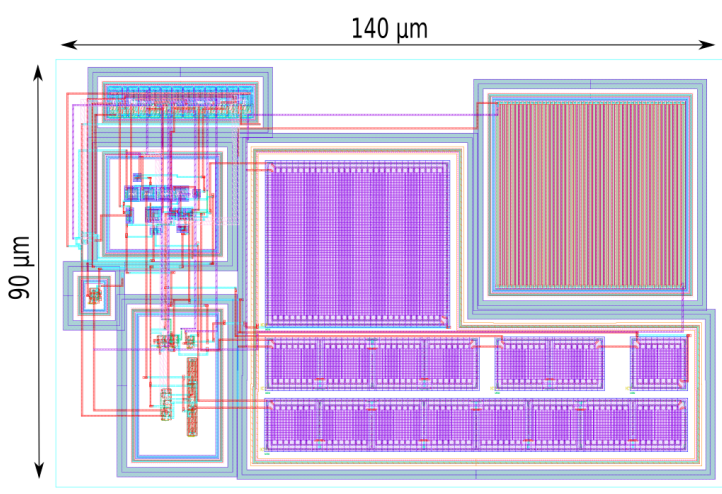

Fig. 4. Layout. 
The typical bias current as well as all the branch current through $M_{b 1}, M_{b 2}, M_{1}$ or $M_{5}$ is $1 \mu \mathrm{A}$. In order to stabilize the amplitude of the triangle waveform the bias current should have inverse temperature and process dependency as the resistor $R$. This is not a problem as the bias current $I_{\text {bias }}$ distributed across the chip is usually derived from a trimmed bandgap voltage $V_{\mathrm{bg}}$ and a reference resistor $R_{\text {bias }}$ as $I_{\text {bias }}=V_{\mathrm{bg}} / R_{\text {bias }}$. If $R_{\text {bias }}$ is the same resistor type as $R$ then the triangle waveform amplitude is a scaled copy of the bandgap voltage.

The transistor dimensions are summarized in Tab. 2 . The gate lengths of the transistors operating as switches were kept at minimum of the given technology at $180 \mathrm{~nm}$. However, the transistors operating as current sources have gate lengths in the order of micro meters for high output resistance and good matching. The former has impact on the triangular waveform linearity and the latter on statistical duty cycle variations.

Figure 4 shows a layout of the proposed circuit (excluding reference generators of Fig. 2b). The circuit takes $0.0126 \mathrm{~mm}^{2}$ out of which the largest part is taken by the capacitors and the resistor.

\section{Simulation Results}

The designed circuit has been simulated in Eldo simulator from Mentor Graphics. The bias current was derived from a constant voltage source and an $\mathrm{N}+$ polysilicon resistor to simulate chip bias current behavior and to stabilize amplitude of the triangle waveform across corners and temperature. The simulated transient waveforms of the main circuit including the reference generators are depicted in Fig. 5. It can be seen that the triangular waveform generated on the cap node exceeds the ideal boundaries given by the reference voltages $V_{\text {low }}$ and $V_{\text {high }}$ (waveforms low and high). This is caused by the propagation delays of the comparator $t_{d}^{+}$and $t_{d}^{-}$as discussed in Sec. 2. The origin of the propagation delays can be seen on the depicted waveforms of internal nodes of the comparator $c m p 1$ and $c m p 2$ which show slew rate limitation caused by the designed constant current of $M_{5}$ and $M_{9}$. The square waveform output $c l k$ is then produced by reshaping the signal on node cmp 2 by the digital CMOS buffer. The oscillating frequency for a typical corner is $0.94 \mathrm{MHz}$, read from the waveforms $t_{d}^{+}$is $3 \mathrm{~ns}$ and $t_{d}^{-}$is $34 \mathrm{~ns}$ out of which $t_{\text {slew }}$ is about $28 \mathrm{~ns}$. We can compare this result with a theoretical value given by (6). Using (values from operating point analysis) $V_{D D}=1.8 \mathrm{~V},\left|V_{\mathrm{THP}}\right|=0.55 \mathrm{~V}, V_{d s 6}^{\text {sat }}=0.19 \mathrm{~V}$, $C_{p}=4.9 \mathrm{fF}, g_{m 6}=18 \mu \mathrm{S}$ and $\mathrm{SR}^{-}=1 \mathrm{~V} / \mu$ s we get theoretical value of $t_{\text {slew }}$ equal to $24 \mathrm{~ns}$ which is in good agreement with the simulated value.

The average current consumption of the generator core (w/o ref. gens. on Fig. 2b) is $4.1 \mu \mathrm{A}$. This is equivalent to $7.38 \mu \mathrm{W}$ at the respective supply voltage.

In order to evaluate process spread of the circuit a Monte Carlo (MC) analysis was run on top of the transient simulation. Further to assess the effectiveness of the trimming
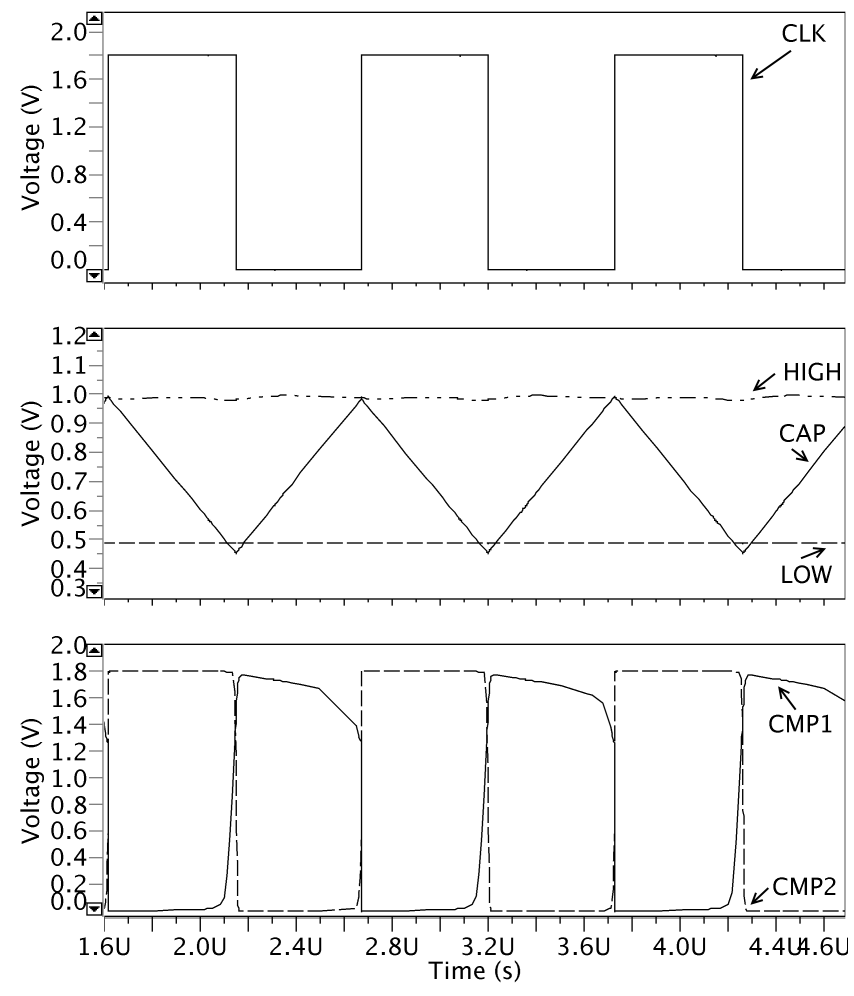

Fig. 5. Simulation results.

method a transient sweep across all the trimming steps was simulated in each MC run and the value closest to the target value (i.e. $1 \mathrm{MHz}$ in this case) was selected. This in fact simulates a real factory trimming.

Figures 6 and 7 shows histograms and statistical parameters of 500 runs of MC analysis. Results of oscillation frequency before trimming can be seen in Fig. 6a. The maximum deviation from the nominal frequency is $26 \%$ and is caused by the process variability of sheet capacitance and sheet resistance in the given technology process. Figure $6 b$ shows histogram of frequency after trimming. The maximum deviation is now $4.27 \%$ from the nominal frequency. Duty-cycle variation histogram is in Fig. $6 \mathrm{c}$ and its standard deviation is $0.82 \%$. This statistical variation of the duty-cycle is caused by the mismatches of current mirrors $M_{b 3}-M_{4}$ and $M_{b 2}-M_{1}$ and can be improved by enlarging the area of the transistors [14].

Figure 7 shows histogram of the peak-to-peak amplitude of the triangle waveform. As described above, the bias current was assumed to be derived from an ideal bandgap voltage reference and the same resistor type as resistor $R$. The amplitude of the triangle waveform is thus not affected by the process spread of the resistor (which is around $\pm 20 \%$ ) and is given mostly by the mismatches of $M_{b 1}, M_{5}$ and $R$.

Variation of the oscillation frequency with temperature can be see in Fig. 8. For the extended temperature range spanning from $-40^{\circ} \mathrm{C}$ to $125^{\circ} \mathrm{C}$ the total frequency variation is $\pm 1.05 \%$ and the temperature coefficient is therefore $127 \mathrm{ppm} /{ }^{\circ} \mathrm{C}$. 

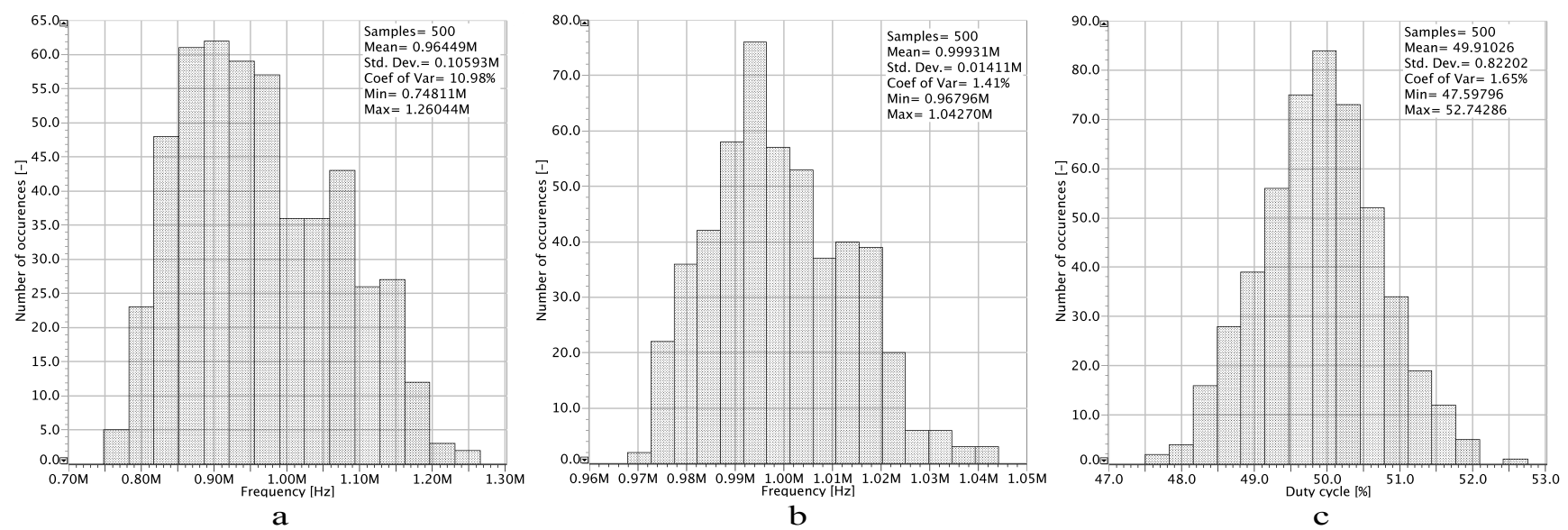

Fig. 6. Monte Carlo analysis histograms: (a) Frequency before trimming, (b) frequency after trimming, (c) duty cycle.

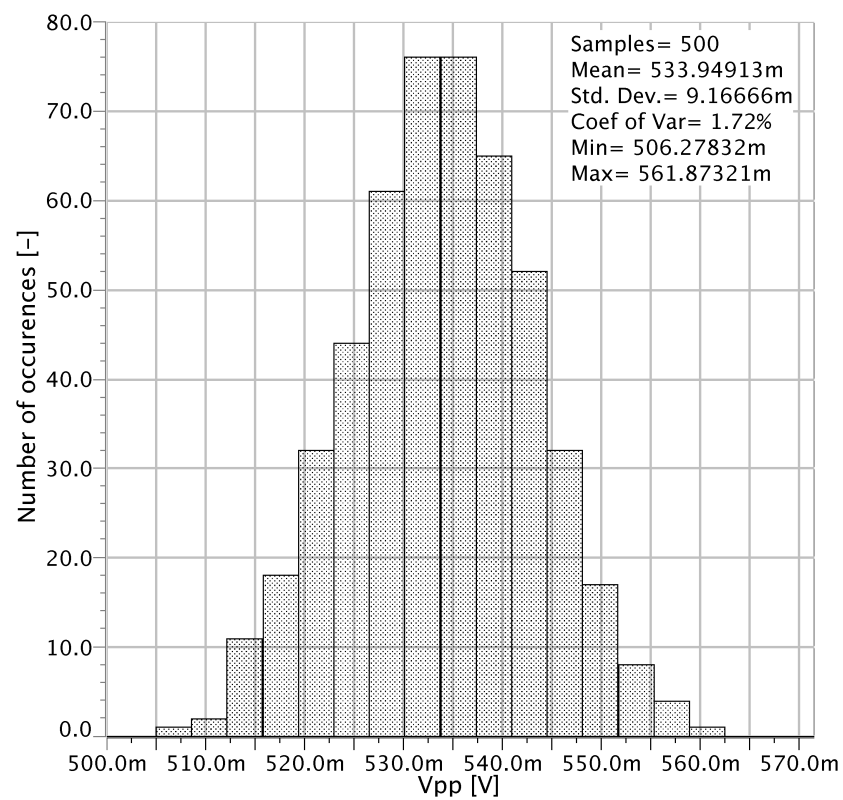

Fig. 7. Triangle waveform peak-to-peak histogram.

\section{Conclusion}

A new area efficient circuit generating triangular waveform was proposed. Oscillating period together with the major source of error caused by the propagation delay of the comparator was derived. A $1-\mathrm{MHz}$ waveform generator based on the proposed topology was designed in STMicroelectronics 180-nm BCD technology consuming 7.38 $\mu \mathrm{W}$ and occupying only $0.0126 \mathrm{~mm}^{2}$. The temperature and process stability of the oscillation frequency depends on the resistors and capacitors available in the given technology. The type of these elements can be selected to at least partially compensate for the temperature behavior of each other. In the presented design a temperature coefficient of $127 \mathrm{ppm} /{ }^{\circ} \mathrm{C}$ was achieved. To cope with the process spread a trimming is usually employed as was demonstrated. The proposed topology can be used as a general purpose square wave generator or as a triangular generator in DC-DC converters.

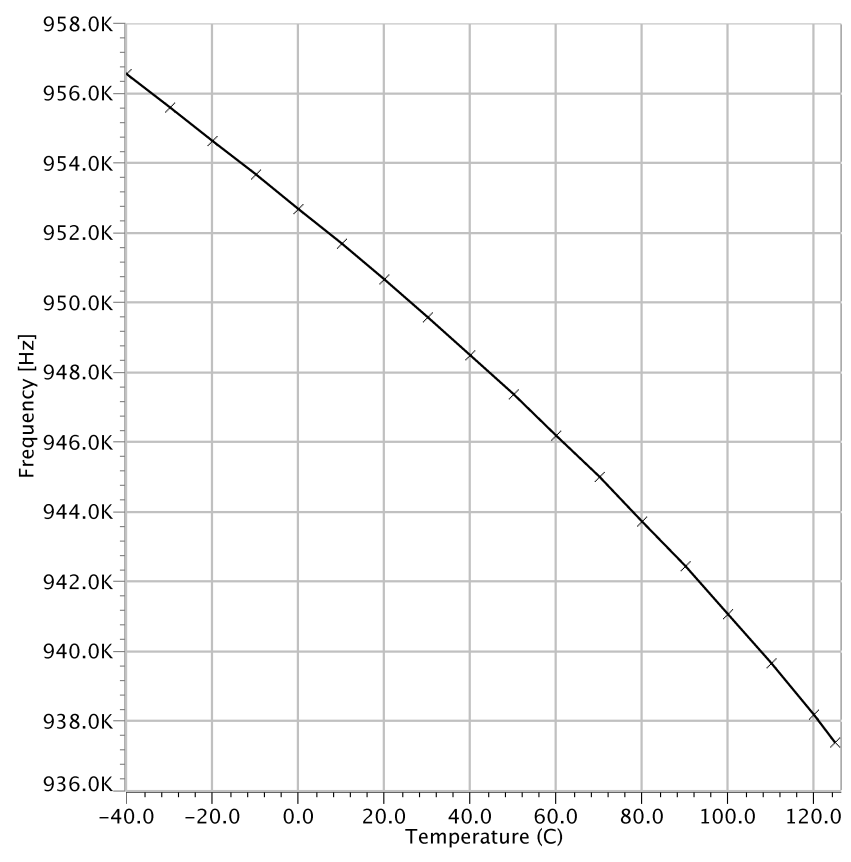

Fig. 8. Frequency temperature variation.

\section{Acknowledgments}

The work has been supported by the grant No. SGS12/143/OHK3/2T/13 of the CTU in Prague.

\section{References}

[1] HUANG, K.-K., WENTZLOFF, D. D. A 1.2-MHz 5.8$\mu \mathrm{W}$ temperature-compensated relaxation oscillator in 130-nm CMOS. IEEE Transactions on Circuits and Systems II: Express Briefs, 2014, vol. 61, no. 5, p. 334-338. ISSN: 1549-7747. DOI: $10.1109 /$ TCSII.2014.2312634

[2] CHIANG, Y.-H., LIU, S.-I. A submicrowatt 1.1-MHz CMOS relaxation oscillator with temperature compensation. IEEE Transactions 
on Circuits and Systems II: Express Briefs, 2013, vol. 60, no. 12, p. 837-841. ISSN: 1549-7747. DOI: 10.1109/TCSII.2013.2281920

[3] BEHJATI, H., NIU, L., DAVOUDI, A., et al. Alternative timeinvariant multi-frequency modeling of PWM DC-DC converters. IEEE Transactions on Circuits and Systems I: Regular Papers, 2013, vol. 60, no. 11, p. 3069-3079. ISSN: 1549-8328. DOI: $10.1109 /$ TCSI.2013.2252641

[4] RAMIREZ-ANGULO, J. A compact current controlled CMOS waveform generator. IEEE Transactions on Circuits and Systems II: Analog and Digital Signal Processing, 1992, vol. 39, no. 12, p. 883-885. ISSN: 1057-7130. DOI: 10.1109/82.208587

[5] BERNARD, S., AZAIS, F., BERTRAND, Y., et al. A high accuracy triangle-wave signal generator for on-chip ADC testing. In Proceedings of the Seventh IEEE European Test Workshop, 2002, p. 89-94. DOI: 10.1109/ETW.2002.1029644

[6] GARIMELLA, A., KALYANI-GARIMELLA, L. M., ROMERO, R., et al. New compact and versatile wide tuning range CMOS voltage controlled oscillator. In 48th Midwest Symposium on Circuits and Systems, 2005, p. 515-518. DOI: 10.1109/MWSCAS.2005.1594151

[7] CHUNG, W.-S., CHA, H.-W., KIM., H.-J. Triangular/squarewave generator with independently controllable frequency and amplitude. IEEE Transactions on Instrumentation and Measurement, 2005, vol. 54, no. 1, p. 105-109. ISSN: 0018-9456. DOI: $10.1109 /$ TIM.2004.840238

[8] PAL, D., SRINIVASUlu, A., PAL, B. B., et al. Current conveyor-based square/triangular waveform generators with improved linearity. IEEE Transactions on Instrumentation and Measurement, 2009, vol. 58, no. 7, p. 2174-2180. ISSN: 0018-9456. DOI: 10.1109/TIM.2008.2006729

[9] SILAPAN, P., SIRIPRUCHYANUN, M. Fully and electronically controllable current-mode Schmitt triggers employing only single MOCCCDTA and their applications. Analog Integrated Circuits and Signal Processing, 2011, vol. 68, no. 1, p. 111-128. ISSN: 1573-1979. DOI: $10.1007 / \mathrm{s} 10470-010-9593-2$

[10] CHIEN, H.-C. Voltage-controlled dual slope operation square/triangular wave generator and its application as a dual mode operation pulse width modulator employing differential voltage current conveyors. Microelectronics Journal, 2012, vol. 43, no. 12 p. 962-974. ISSN: 0026-2692. DOI: 10.1016/j.mejo.2012.08.005
[11] SOTNER, R., JERABEK, J., HERENCSAR, N. Voltage differencing buffered/inverted amplifiers and their applications for signal generation. Radioengineering, 2013, vol. 22, no. 2, p. 490-504. ISSN: $1805-9600$

[12] SOTNER, R., JERABEK, J., HERENCSAR, N., et al. Design of Z-copy controlled-gain voltage differencing current conveyor based adjustable functional generator. Microelectronics Journal, 2015, vol. 46, no. 2, p. 143-152. ISSN: 0026-2692. DOI: $10.1016 /$ j.mejo.2014.11.008

[13] JERABEK, J., SOTNER, R., DOSTAL, T., et al. Simple resistorless generator utilizing Z-copy controlled gain voltage differencing current conveyor for PWM generation. Elektronika ir Elektrotechnika, 2015, vol. 21, no. 5, p. 28-34. ISSN: 1392-1215. DOI: $10.5755 /$ j01.eee.21.5.13322

[14] PELGROM, M. J., DUINMAIJER, A. C., WELBERS, A. P. Matching properties of MOS transistors. IEEE Journal of Solid-State Circuits, 1989, vol. 24, no. 5, p. 1433-1439. ISSN: 0018-9200. DOI: $10.1109 /$ JSSC.1989.572629

\section{About the Authors ...}

Martin Ď̌íNOVSKÝ was born in 1985. He received his Master's degree from the Czech Technical University in Prague and is currently studying towards Ph.D. at the Department of Circuit Theory. He is with STMicroelectronics since 2010 working as a senior design engineer. His research interests include analog and mixed-signal circuits, IP modeling and verification methodologies.

Jiří HOSPODKA was born in 1967. He received his Master's and Ph.D. degrees from the Czech Technical University in Prague in 1991 and 1995, respectively. Since 2007 he has been working as associate professor at the Department of Circuit Theory at the same university. Research interests: circuit theory, analogue electronics, filter design, switchedcapacitor, and switched-current circuits. 